\title{
Molecular Characterization of Human Adenoviruses in Children Suffered From Acute Gastroenteritis By Partial Hexon Region
}

\author{
Research Article
}

Rambha Tripathi ${ }^{1,2}$, Vani Gupta ${ }^{2}$, Divya Gupta ${ }^{1,2}$, Dharam Veer Singh ${ }^{1}$, Pooja Gaur ${ }^{1}$, Ravish Katiyar ${ }^{1}$, Shally Awasthi ${ }^{2}$, Piyali Bhattacharya ${ }^{1}$, Tapan N Dhole $^{1^{*}}$

${ }^{1}$ Sanjay Gandhi Postgraduate Institute of Medical Sciences, Lucknow, Uttar Pradesh, India.

${ }^{2}$ King George's Medical University, Lucknow, Uttar Pradesh, India.

\section{Abstract}

Introduction: Viral gastroenteritis is an important cause of childhood morbidity and mortality, in developing countries. Human adenovirus (HAdV) poses a major risk in children, elderly people and immunocompromised persons, causes acute diarrhea at irregular intervals as well as in outbreaks.

Aim: The study was aimed to investigate the molecular characterization and associated clinical features of HAdV infection in North Indian children affected with acute gastroenteritis (AGI) infection.

Materials and Methods: HAdV has been identified by PCR, targeting partial hexon gene from fecal specimen of 250 AGI affected children.

Results: A total 14 (5.6\%) HAdV positives were confirmed by PCR, among them 8 males $(3.2 \%)$ and $6(2.4 \%)$ were females. Maximum positivity was $57.14 \%$ in the age group of $0-5$ years AGI affected children. HAdV-B was the most prevalent followed by C, D, F and A in North Indian children, out of them serotype B3 (28.57\%) was prevalent, followed by C2, C5 and F41 (14.29\% each); A12, B34, D63 and D28 (7.14\% each).

Conclusion: This study contributes to a better understanding about HAdV that might involve in AGI illnesses in young children, prognosis helps in planning and prevention from HAdV infection. HAdV-B3 was the most common adenovirus serotypes circulating in the North India. To the best of our knowledge, this is the first study on molecular characterization of HAdV strains from North Indian AGI children.

Keywords: AGI; HAdV; PCR; Hexon Gene; Phylogenetic Analysis.

Abbreviations: AGI: Acute Gastroenteritis; HAdV: Human Adenovirus; KGMU: King George Medical University; PCR: Polymerase Chain Reaction; SGPGIMS: Sanjay Gandhi Post Graduate Institute of Medical Sciences; WHO: World Health Organization.

\section{Introduction}

Viral gastroenteritis is an important cause of childhood morbidity and mortality, in developing countries [1]. Every year 2.5 million deaths are estimated to occur due to enteric infections, greatly impacting children younger than five years of age [2]. Human adenovirus (HAdV) creates a major risk in children, elderly people and immunocompromised persons [3]. It causes acute diarrhea at irregular intervals as well as in outbreaks [4]. HAdV can cause a broad range of human diseases such as acute respiratory tract infection (ARTI), pneumonia, bronchitis, conjunctivitis, hepatitis, ocular infection, hemorrhagic cystitis, gastroenteritis, GI and urinary tract infection (UTI). HAdV infectivity is transmitted by inhalation and direct contact with small droplet aerosols or the fecal-oral route [5].

HAdV discovered in 1953 and its prevalence is approximately $4-12 \%$ in acute gastroenteritis (AGI) and ARTI [6]. It belongs to the family Adenoviridae and genus Mastadenovirus and these are non-enveloped, icosahedral viruses. Adenoviruses have linear double-stranded DNA that generally range from 26 to $45 \mathrm{~kb}$ and

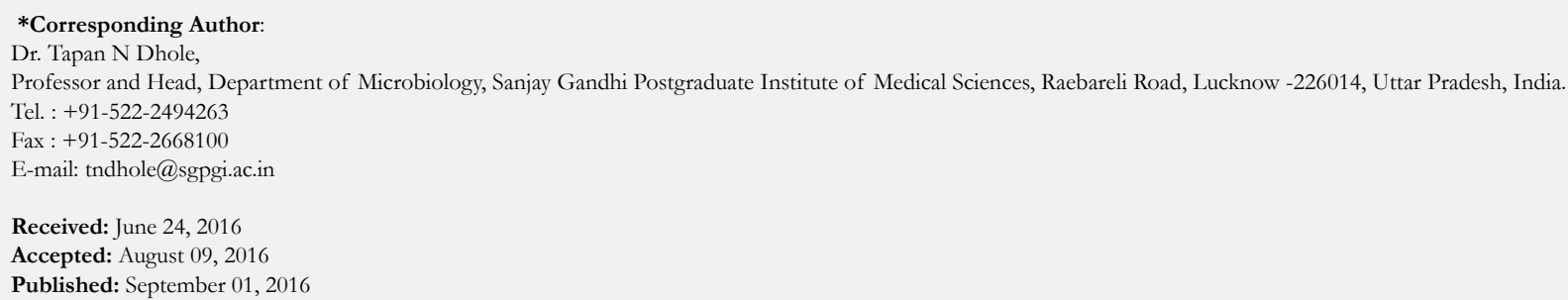

Citation: Tapan N Dhole, et al., (2016) Molecular Characterization of Human Adenoviruses in Children Suffered From Acute Gastroenteritis By Partial Hexon Region. Int J Virol Stud Res. 4(4), 42-47. doi: http://dx.doi.org/10.19070/2330-0027-160007

Copyright: Tapan N Dhole ${ }^{\circ}$ 2016. This is an open-access article distributed under the terms of the Creative Commons Attribution License, which permits unrestricted use, distribution and reproduction in any medium, provided the original author and source are credited. 
are encapsulated in an icosahedral protein shell, particles ranges from $70-90 \mathrm{~nm}$ in size. Based on genomic studies, 68 types of HAdV have been recently classified, which can be divided into seven different species (A-G) [7,8]. Virus was detected by PCR using degenerate primers targeting highly conserved region of partial hexon gene [9] and characterized by sequencing and phylogenetic analysis.

So, the present study planned for molecular characterization of HAdV in children suffering from AGI by PCR method of hexon gene and also to determine species and serotypes of HAdV that are circulating in North Indian children.

Casas et al., (2005), developed and validated a novel methods for species and serotypes by phylogenetic analysis using multiple sequence alignment with some reference sequences represents each known serotypes in data base [9]. We also use some reference sequence and method to assign species and serotypes of our unknown sequence.

\section{Materials and Methods}

\section{Sample collections}

A total 250 clinically diagnosed cases of AGI in children of age group less than 15 years were recruited in the study. Fecal specimens were collected after obtaining informed consent from Department of Microbiology, SGPGIMS Lucknow and Department of Pediatrics, KGMU, Lucknow, Uttar Pradesh, India, during the March 2012 to September 2013. In 2012, we have collected 123 stool specimen and 127 fecal specimens in 2013. The study protocol was approved by the Ethics Committee of SGPGIMS and KGMU Lucknow. We have collected 15-20 ml liquid of watery stool and one sample per patient in a clean and dry screw capped, wide mouth plastic container. Samples were immediately transported (maintaining the cold chain) and stored at $-20^{\circ} \mathrm{C}$ for further analysis.

\section{Processing of Gastroenteritis Samples}

For viral detection stools were processed as per WHO guidelines [10]. Briefly, Stool suspension (10\%) was prepared in 0.01 $\mathrm{M}$ phosphate buffered saline (PBS) (PH 7.2) in a biosafety cabinet level II and vortex at $300 \mathrm{rpm}$ for 20 minutes followed by centrifugation at $3000 \mathrm{rpm}$ for 30 minutes at $4^{\circ} \mathrm{C}$. Leaved for 10 minutes at room temperature (RT) and supernatant was stored at $-20^{\circ} \mathrm{C}$ for further analysis.

\section{DNA Extraction}

Viral DNA extracted from $200 \mu \mathrm{l}$ stool supernatant using the QIAmp, DNA extraction mini kit (Qiagen, Valencia, CA), according to manufacturer's instructions. DNA was stored at $-80^{\circ} \mathrm{C}$ for further identification.

\section{PCR for Detection of Adenovirus}

For the amplification and detection of $\mathrm{HAdV}$, we used $5 \mu \mathrm{l}$ of DNA in $45 \mu$ l of reaction mixture containing $10 \mathrm{mM}$ Tris-HCL (pH8.3), $50 \mathrm{mM} \mathrm{KCL}, 500 \mathrm{mM}$ (each) dNTPs, $4 \mathrm{mM} \mathrm{MgCl}_{2}, 0.5$ $\mu \mathrm{l}$ of Taq polymerase $(5 \mathrm{U} / \mu \mathrm{l})$ and $20 \mathrm{pmol}$ of the generate for- ward 5'- CAACACCTAYGASTACATGAA-3' and reverse primer 5'-KATGGGGTARAGCATGT'T-3' with PCR having initial denaturation step at $94^{\circ} \mathrm{C}$ for 5 minutes, denaturation at $94^{\circ} \mathrm{C}$ for 1 minute, annealing at $50^{\circ} \mathrm{C}$ for 1 minute, extension at $68^{\circ} \mathrm{C}$ for 1 minute for 30 cycles and a final extension at $68^{\circ} \mathrm{C}$ for $5 \mathrm{~min}-$ utes [9]. The amplified product of $475 \mathrm{bp}$ was visualized on $2 \%$ agarose gel pre-stained with ethidium bromide along with molecular weight marker VIII (Sigma-Aldrich) under ultraviolet light, imaged with Gel Doc XR System (Bio-Rad, Hercules, CA). The PCR products were commercially sequenced by Sanger sequencing method.

\section{Sequence and Phylogenetic Analysis}

Molecular identification of each serotype was done by pair-wise comparison of the partial hexon amplicon sequence with database of all HAdV serotypes using the BLAST program (blast. ncbi.nlm.nih.gov/Blast.cgi) from Gene bank. The sequence data were aligned by Clustal W multiple sequence alignment tool. The evolutionary history was inferred by the neighbour-joining method, based on the Kimura 2-parameter model and 1000 bootstrap replications using the MEGA 6 software program (www.megasoftware.net) showed in Figure 4.

\section{Results}

In this study, we have analyzed 250 AGI fecal specimens (Males =117; Females=133), median age was 84 months (minimum 3 months, max. 180 months). A total 14 (5.6\%) specimens were found positive for HAdV (adeno positive band showed in Figure $1)$, among them 8 male $(3.2 \%)$ and $6(2.4 \%)$ were female pediatric patients with median age was 38 months (minimum 3 months, max. 158 months), showed in Table 1 . Maximum positivity was $57.14 \%$ in the age group of $0-5$ years, while equal numbers of positivity $(21.43 \%)$ were observed in each age group; $5-10$ years and $10-15$ years. HAdV-C $(37.5 \%)$ was the most common species in the age group of $0-5$ years followed by B (25\%), A, D and F $(12.5 \%)$, whereas in age group 5-10 years the HAdV-B (25\%) were frequent followed by species D $(12.5 \%)$. In higher age group of 10-15 years species B, C and F (12.5\%) were equally represented (Figure 2). HAdV-B was the most prevalent followed by C, D, F and $\mathrm{A}$ in North Indian children.

\section{Serotyping}

Serotyping has been done by phylogenetic analysis taking reference sequences representing each serotype [9]. HAdV-B3 (28.57\%) was prominent serotype, followed by C2, C5, F41 (14.29\% each), A12, B34, D63 and D28 (7.14 \% each) showed in Figure 3. The percentage of HAdV infection per year calculated in 2012 and 2013 was $71.43 \%$ and $28.57 \%$ respectively. The HAdV infection was most prevalent in 2012. Overall, HAdV infection was recorded higher during the spring season peak in month February to March and in rainy season May to September. General clinical features of AGI in the study participants were; watery stool, high fever, vomiting, abdominal pains and dehydration. Among the participants that tested positive for adenovirus, the recorded clinical manifestations are presented in Table 2 . HAdV positive patient's details, including age/gender, main clinical symptoms and identified serotypes are showed in Table 3 . 
Figure 1. Gel electrophoresis image for HAdV positive isolates

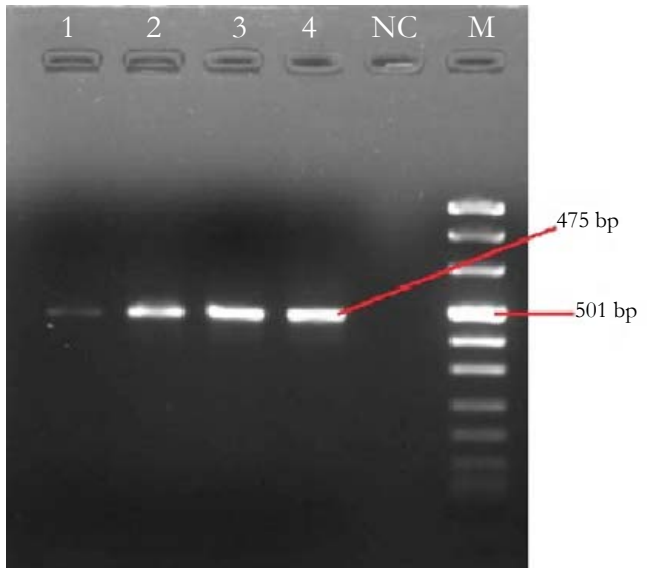

Lane 1 to 4 Human adenovirus positive band; Lane 5 Negative control; Lane 6 DNA molecular weight marker VIII (SIGMA).

Figure 2 HAdV positivity in different age groups

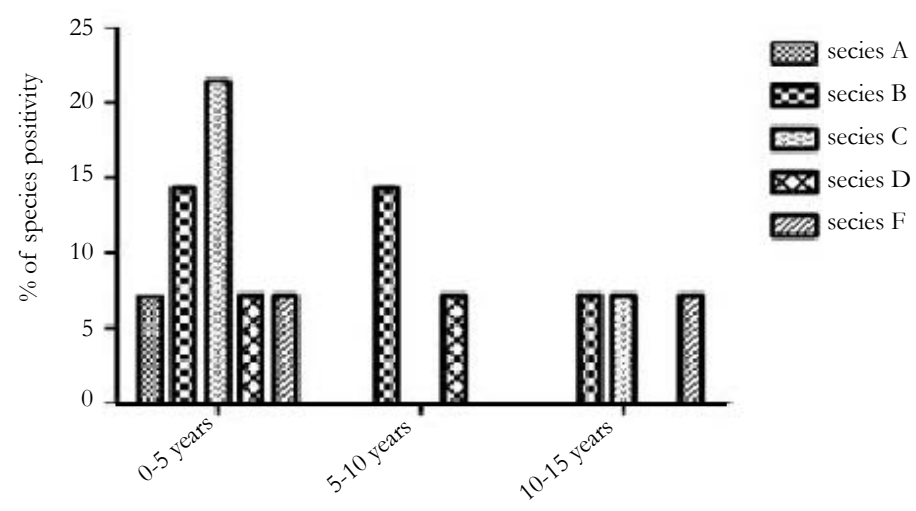

Figure 3. Distribution of HAdV serotypes.

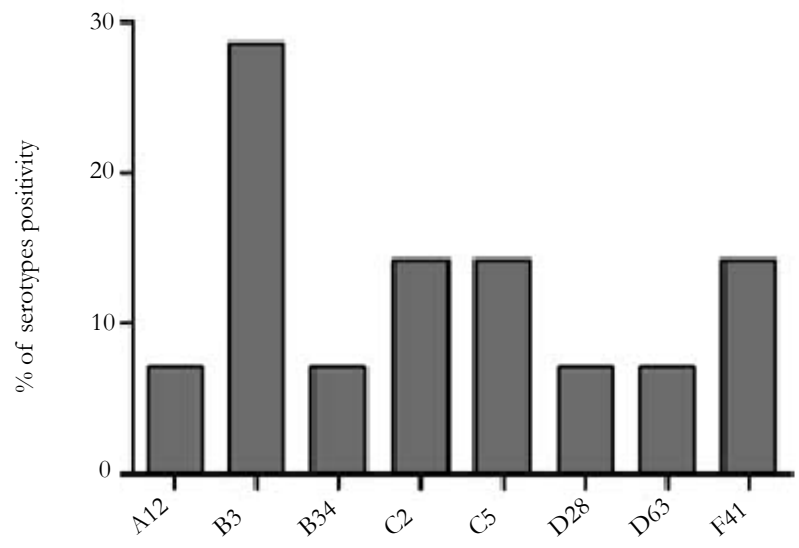

Phylogenetic analysis identified 97 to $99 \%$ similarities of strains with other circulating strains in different parts of India as well as in other countries showed in Table 4 (and Figure 4).

\section{Discussion}

HAdV is most commonly associated with AGI illnesses. Young children and immunocompromised patients are especially vulnerable to severe complications of HAdV infection [11, 12]. Our data shows that, during the study period (March 2012 to September 2013), the highest rate of HAdV infection occurred in 2012. The seasonal distribution of HAdV infection shows a higher prevalence in spring season (42.86\%; February and March) and in rainy season (57.14\%; May to September), which is similar to Dhaka city, Bangladesh 2009 [13].

In the present study the rate of HAdV infection was found $5.6 \%$ which is higher than previous similar studies, 3.1\% in Tunisia [14], $1.9 \%$ in Dhaka city, Bangladesh [13] and lower than Egypt; $10.4 \%$ [15], Ghana; 19.8\% [16], in northwest Nigeria; 23\% [17], Albania $23.2 \%$ [3], and $37.4 \%$ in Kenya [18]. Male/female ratio (M/F) of $\mathrm{HAdV}$ infected pediatric children were 1.33:1, similar to Taiwan 1.30:1 [19], where as HAdV infection is higher in Israel 1.48:1 [20]. 
Table 1. HAdV prevalence, distribution of gender and age group.

\begin{tabular}{|c|c|c|}
\hline Demographic & \multicolumn{2}{|c|}{ Gastroenteritis children } \\
\hline & $\begin{array}{c}\text { Human adenovirus } \\
\text { Positive sample } \\
\mathbf{n = 1 4} \mathbf{( 5 . 6 \% )}\end{array}$ & $\begin{array}{c}\text { Human adenovirus } \\
\text { negative sample } \\
\mathbf{n = 2 3 6} \mathbf{( 9 4 . 4 \% )}\end{array}$ \\
\hline Gender & & $109(46.19 \%)$ \\
\hline Male & $08(3.2 \%)$ & $127(53.81 \%)$ \\
\hline Female & $06(2.4 \%)$ & 84 \\
\hline Age (month) & & 132 \\
\hline Median & 38 & $76(32.20 \%)$ \\
\hline Mode & 3 & $71(30.09 \%)$ \\
\hline Age Group in Year & & $89(37.71 \%)$ \\
\hline $0-5$ Years & $08(57.14 \%)$ & \\
\hline $5-10$ Years & $03(21.43 \%)$ & \\
\hline $10-15$ Years & $03(21.43 \%)$ & \\
\hline
\end{tabular}

Table 2. Clinical manifestations in children positive for adenovirus diarrhea among under-15 year's children.

\begin{tabular}{|c|c|}
\hline Signs $/$ Symptoms & No. of Human adenovirus Positive $(\mathbf{n}=\mathbf{1 4})$ \\
\hline Watery stools & $14(100 \%)$ \\
\hline Low fever $\left(99.2^{\circ} \mathrm{F}\right)$ & $04(28.57 \%)$ \\
\hline Abdominal pain & $06(42.86 \%)$ \\
\hline High Fever $\left(103^{\circ} \mathrm{F}\right)$ & $05(35.71 \%)$ \\
\hline Mild dehydration & $02(14.29 \%)$ \\
\hline Dehydration & $03(21.43 \%)$ \\
\hline Vomiting & $11(78.57 \%)$ \\
\hline Stool with mucus & $0(0 \%)$ \\
\hline
\end{tabular}

Table 3. Summary of HAdV-positive patients data, including age and main symptoms of Gastroenteritis together with HAdV typing results.

\begin{tabular}{|c|c|c|c|c|}
\hline S.no. & Patient ID & Age/Gender & Signs/ Symptoms & Serotypes \\
\hline 1 & Ad-ARP-2G & 40 months/Male & Watery stool, low fever and abdominal pain. & A-12 \\
\hline 2 & Ad-ARP-6G & 71 months/FeMale & Watery stool, vomiting and dehydration. & B-3 \\
\hline 3 & Ad-ARP-10G & 29 months/Male & Watery stool with high fever. & C-5 \\
\hline 4 & Ad-ARP-19G & 36 months/FeMale & Watery stool, vomiting, dehydration, low fever and abdominal pain. & B-3 \\
\hline 5 & Ad-ARP-23G & 14 months/FeMale & Watery stool, vomiting with high fever and dehydration. & B-3 \\
\hline 6 & Ad-ARP-28G & 18 months/Male & Watery stool with mucus, vomiting, high fever and abdominal pain. & F-41 \\
\hline 7 & Ad-ARP-39G & 3 months/FeMale & Watery stool, vomiting, high fever and abdominal pain. & C-2 \\
\hline 8 & Ad-ARP-42G & 67 months/Male & Watery stool, mild dehydration and vomiting with high fever. & B-3 \\
\hline 9 & Ad-ARP-57G & 16 months/Male & Watery stool and vomiting. & C-5 \\
\hline 10 & Ad-ARP-69G & 158 months/FeMale & Watery stool, vomiting, low fever and abdominal pain. & F-41 \\
\hline 11 & Ad-ARP-95G & 12 months/Male & Watery stool, vomiting. & D-63 \\
\hline 12 & Ad-ARP-126G & 144 months/Male & Watery stool and vomiting. & C-2 \\
\hline 13 & Ad-ARP-127G & 132 months/Male & Watery stool, low fever, mild dehydration and abdominal pain. & B-34 \\
\hline 14 & Ad-ARP-140G & 117 months/FeMale & Watery stool and vomiting. & D-28 \\
\hline
\end{tabular}


Figure 4. Phylogenetic tree of adenovirus hexon gene sequences of fecal specimens and other reference strain. The phylogenetic trees were constructed by the neighbour-joining method with 1,000 bootstrap replications in the Clustal $\mathrm{W}$ program.

Bootstrap proportions (1,000 replications) are indicated as a percentage in each node. A number of identical nucleotide sequences were presented in parentheses.

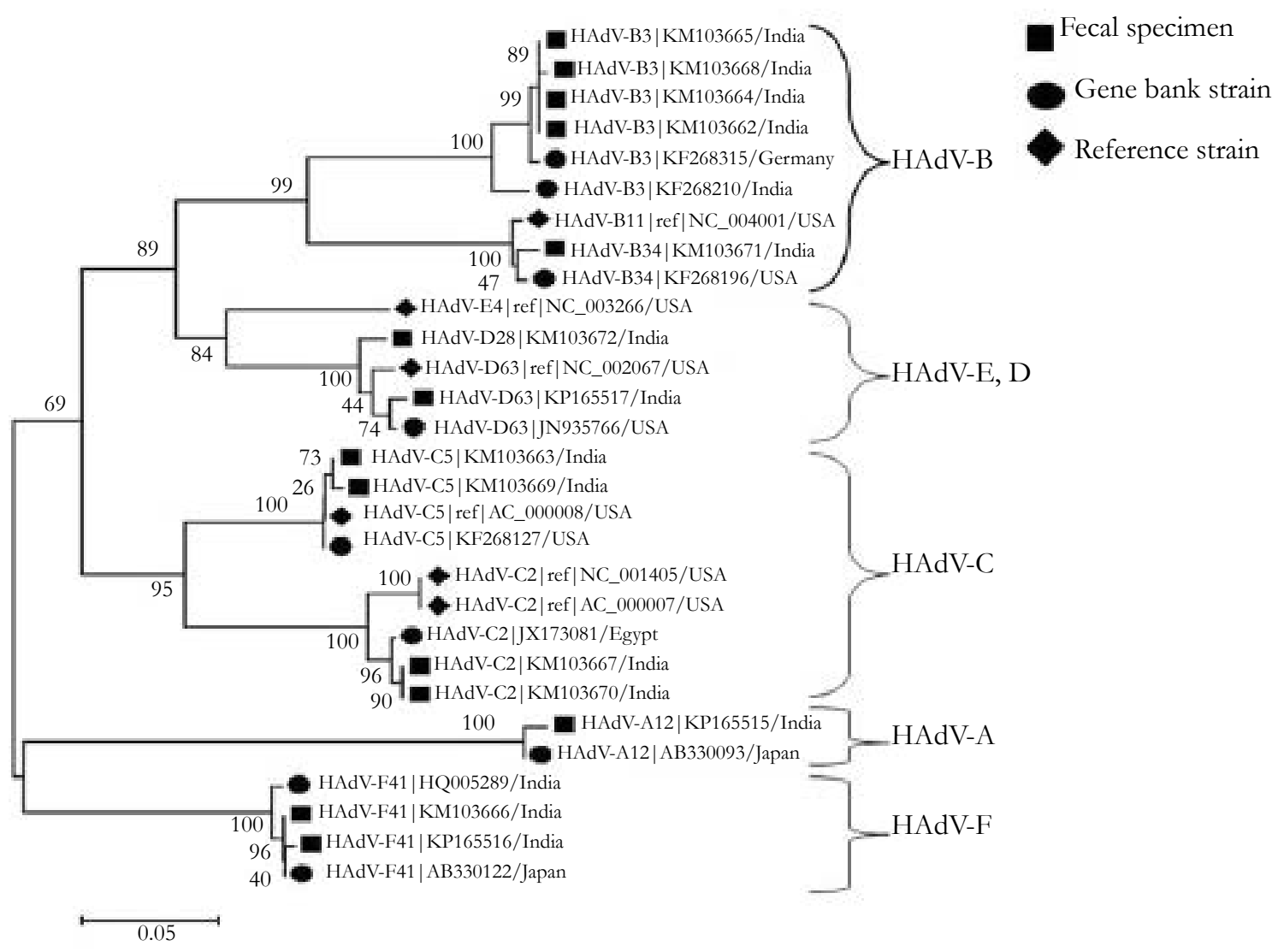

Table 4. Human adenovirus (HAdV) reference strains from the Genbank used for the typing of HAdV isolates from North India.

\begin{tabular}{|c|c|c|}
\hline Accession Number & Serotypes & Country \\
\hline AB330093 & A12 & Japan \\
\hline KF268315 & B3 & Germany \\
\hline KF268210 & B3 & India(Tamilnadu) \\
\hline AB330122 & F41 & Japan \\
\hline HQ005289 & F41 & India (Kolkata) \\
\hline JX173081 & C2 & Egypt \\
\hline KF268127 & C5 & USA \\
\hline KF268196 & B34 & USA \\
\hline JN935766 & D63 & USA \\
\hline KF268320 & D28 & Germany \\
\hline
\end{tabular}

According to their age group, 0-5 years of infants and children the higher rates of HAdV infection was $57.14 \%$ which is similar to other studies of HAdV infections worldwide [20]. Cooper et al., demonstrated that, children had been infected early in life, had acquired immunity to this infection [21]. Species B shows the consistent results $25 \%$ positivity in age groups $0-5$ years and $5-10$ years of HAdV affected children, but the result varies in the age group 10-15 years children, species B shows the low positivity $12.5 \%$.
The sequence based analysis of the partial hexon gene showed the presence of HAdV serotypes A12, B3, B34, C2, C5, D28, D63 and F41 in AGI infected children [22-25]. HAdV-B and C species shows higher prevalence followed by species $\mathrm{D}, \mathrm{F}$ and $\mathrm{A}$ respectively. We have investigated the HAdV-B3 predominant genotype prevalent in the year 2012, and our findings was supported by China published in 2012 [26, 27]. HAdV-B3 genotype was most prevalent and the results is supported by previous study by Yeung $\mathrm{R}$ et al., [28] in Canadian Population. Very few studies have been 
published from India in which they have mostly found the A12, B3, C2, C5, F40 and F41 HAdV serotypes [29, 30]. A study from Chennai, India found the HAdV B3 serotypes [29]. A study from Chicago shows the diarrhea causing HAdV are known to be associated with AGI in children [31]. As seen in our study fever, vomiting, watery stools and dehydration are usually associated features of AGI more so of viral GI.

Phylogenetic analysis reveals that, 97-99\% similar strains are found in India and across the other countries. C5, B34, D63 are most common strains circulating in USA where as B3, D28 strains are common in Germany and B3, F41 are common strains in Tamilnadu and Kolkata (India) respectively. By the phylogenetic analysis we can conclude that, the source country of particular strains are A12 Japan; B3, D28 Germany; B3 Tamilnadu; F41 Japan, Kolkata; C2 Egypt; C5, B34, D63 USA. Further studies are needed to validate our results with long samples sizes in AGI children affected with HAdV infection.

The resulting data is very useful to understand the molecular characterization and seasonal distribution of HAdV infection in North Indian children and this information can be used to take preventive actions for controlling future outbreaks of HAdV.

In conclusion, this study indicates that HAdV-B was the most common adenovirus species circulating in the North Indian children. To the best of our knowledge, this is the first study on molecular characterization of HAdV strains from North Indian AGI children.

\section{Acknowledgements}

This study was supported by an ICMR-SRF fellowship awarded to Mrs. Rambha Tripathi from Indian Council of Medical Research, Government of India, New Delhi (Ref. no. 80/840/2013-ECDI). I acknowledge Ms. Ankita Pandey for technical assistance.

\section{References}

[1]. Hart C, N Cunliffe, O Nakagomi (2009) Diarrhoea caused by viruses. Manson's tropical diseases. (22nd ed.), Philadelphia: Saunders Elsevier, 815-24.

[2]. Girard MP (2006) A review of vaccine research and development: human enteric infections. Vaccine 24(15): 2732-2750.

[3]. La Rosa G (2015) Genetic diversity of human adenovirus in children with acute gastroenteritis, Albania, 2013-2015. BioMed research international, 2015.

[4]. Van R (1992) Outbreaks of human enteric adenovirus types 40 and 41 in Houston day care centers. The Journal of pediatrics 120(4): 516-521.

[5]. Sriwanna, P.( 2013) Molecular characterization of human adenovirus infection in Thailand, 2009-2012. Virology journal. 10(1):1.

[6]. Echavarría M ( 2009) Adenoviruses. eLS,

[7]. Davison, A.J., M. Benkő,B. Harrach ( 2003) Genetic content and evolution of adenoviruses. Journal of General Virology 84(11): 2895-2908.
[8]. Wold W, M Horwitz ( 2007) Adenoviruses. Fields virology 2: 2395-2436.

[9]. Casas I (2005) Molecular identification of adenoviruses in clinical samples by analyzing a partial hexon genomic region. Journal of clinical microbiology 43(12): 6176-6182.

[10]. World Health Organization.(2004) Polio laboratory manual.( 4th edition), Department of Immunization, Vaccines and Biologicals. Geneva, Switzerland.

[11]. Kojaoghlanian T, P Flomenberg, MS Horwitz (2003) The impact of adenovirus infection on the immunocompromised host. Reviews in medical virology 13(3):155-171.

[12]. Walls T, A Shankar, D Shingadia (2003) Adenovirus: an increasingly important pathogen in paediatric bone marrow transplant patients. The Lancet infectious diseases 3(2): 79-86.

[13]. Dey SK (2009) Molecular epidemiology of adenovirus infection among infants and children with acute gastroenteritis in Dhaka City, Bangladesh. Infection, Genetics and Evolution 9(4): 518-522.

[14]. Fodha I (2006) Identification of viral agents causing diarrhea among children in the Eastern Center of Tunisia. Journal of medical virology 78(9):11981203.

[15]. Kamel AH (2009) Predominance and circulation of enteric viruses in the region of Greater Cairo, Egypt. Journal of clinical microbiology 47(4): 10371045.

[16]. Silva PA (2008) Molecular characterization of enteric viral agents from children in northern region of Ghana. Journal of medical virology $80(10)$ : 1790-1798.

[17]. Aminu M (2007) Adenovirus infection in children with diarrhea disease in Northwestern Nigeria. Annals of African medicine 6(4): 168-73.

[18]. Magwalivha M (2010) High prevalence of species D human adenoviruses in fecal specimens from Urban Kenyan children with diarrhea. Journal of medical virology 82(1): 77-84.

[19]. Tsou TP ( 2012) Community outbreak of adenovirus, Taiwan, 2011. Emerg Infect Dis 18(11):1825-1832.

[20]. Mandelboim M (2011) Adenovirus infections in hospitalized patients in Israel: epidemiology and molecular characterization. Journal of clinical microbiology 49(2): 597-601.

[21]. Cooper R (2000) The epidemiology of adenovirus infections in Greater Manchester, UK 1982-96. Epidemiology and infection 125(2): 333-345.

[22]. Ishiko H (2008) Novel human adenovirus causing nosocomial epidemic keratoconjunctivitis. Journal of clinical microbiology 46(6): 2002-2008.

[23]. Sirena D (2005) The nucleotide sequence and a first generation gene transfer vector of species B human adenovirus serotype 3. Virology 343(2): 283-298.

[24]. Pauly M (2015) Adenovirus in Rural Côte DIvoire: High Diversity and Cross-Species Detection. Ecohealth 12(3): 441-452.

[25]. Singh G (2012) Overreliance on the hexon gene, leading to misclassification of human adenoviruses. Journal of virology 86(8): 4693-4695.

[26]. Zou L (2012) Human adenovirus infection in children with acute respiratory tract disease in Guangzhou, China. Apmis 120(8): 683-688.

[27]. Xie L (2012) Two adenovirus serotype 3 outbreaks associated with febrile respiratory disease and pharyngoconjunctival fever in children under 15 years of age in Hangzhou, China, during 2011. Journal of clinical microbiology 50(6): 1879-1888.

[28]. Yeung R (2009) Characterization of culture-positive adenovirus serotypes from respiratory specimens in Toronto, Ontario, Canada: September 2007June 2008. Virology journal 6(1): 1.

[29]. Janani M, J Malathi, H Madhavan (2012) Isolation of a variant human adenovirus identified based on phylogenetic analysis during an outbreak of acute keratoconjunctivitis in Chennai. The Indian journal of medical research. 136(2): 260-4.

[30]. Dey RS (2011) Circulation of a novel pattern of infections by enteric adenovirus serotype 41 among children below 5 years of age in Kolkata, India. Journal of clinical microbiology 49(2): 500-505.

[31]. Christensen ML ( 1989) Human viral gastroenteritis. Clinical microbiology reviews 2(1): 51-89. 